\title{
Resistive-nanoindentation: contact area monitoring by real-time electrical contact resistance measurement
}

\author{
Solène Comby-Dassonneville ${ }^{\mathrm{a}}$, Fabien Volpi ${ }^{\mathrm{a}}$, Guillaume Parry ${ }^{\mathrm{a}}$, Didier Pellerin ${ }^{\mathrm{b}}$, Marc Verdier $^{\mathrm{a}}$ \\ ${ }^{a}$ Univ. Grenoble Alpes, CNRS, Grenoble INP, SIMaP, 38000 Grenoble, France \\ ${ }^{\mathrm{b}}$ Scientec/CSInstruments, 91940 Les Ulis, France
}

Corresponding author: fabien.volpi@ simap.grenoble-inp.fr

\begin{abstract}
In the past decades, efforts have been made to couple nanoindentation with resistive measurements in order to monitor the real-time contact area, as an alternative to the use of traditional analytical models. In this work, a novel and efficient stand-alone method is proposed to compute contact area using resistivenanoindentation of noble metals (bulk or thin films). This method relies on three steps: tip shape measurement, set-up calibration, application to the sample to be characterized. The procedure is applied to nanoindentation tests on a sample with film-on-elastic-substrate rheology and is successfully validated against experimental measurements of the contact area.
\end{abstract}




\section{Introduction}

In the past three decades, efforts have been made to couple instrumented indentation with resistive measurements [1]. This development was driven by several motivations, such as the local monitoring of phase transformation $[2,3,4,5,6,7,8]$, the study of native oxide fracture $[9,10,11]$, the investigation of MEMS operation at small scales $[12,13]$ and the contact area computation during nanoindentation test $[14,15]$. The later point is of particular interest for the determination of the contact area $A_{c}$ which is essential to compute both the sample Young's modulus and the hardness from nanoindentation tests:

The sample Young's modulus is determined using the Sneddon's relation [16]:

$$
S_{c}=\frac{2}{\sqrt{\pi}} E^{*} \sqrt{A_{c}}
$$

with $S_{c}$ the contact stiffness (measured continuously during nanoindentation test) and $E^{*}$ the reduced modulus, expressed as:

$$
E^{*}=\left(\frac{1-v_{\text {tip }}^{2}}{E_{\text {tip }}}+\frac{1-v_{\text {sample }}{ }^{2}}{E_{\text {sample }}}\right)^{-1}
$$

with $v_{t i p}, v_{\text {sample }}, E_{t i p}, E_{\text {sample }}$ the tip and sample Poisson's ratios and moduli, respectively.

$>\quad$ The sample hardness $H$ is computed using Eq. (3), with $L$ the load on sample.

$$
H=\frac{L}{A_{e}}
$$

The contact area $A_{c}$ (defined as the projected area of the contact interface between the tip and the sample) is however complex to monitor, even for the simplest case of homogeneous semi-infinite specimens. This is because contact area depends both on the tip geometry and on the contact depth $h_{c}$ ( $h_{c}$ being the length of the tip effectively in contact with the sample (Supplementary Fig. S1)). The tip geometry is either determined by AFM imaging or by using a calibration sample with well-known mechanical properties [17]. As shown in Supplementary Fig. S1, the contact depth $h_{c}$ depends on sample rheology: the sample 
can either sink-in or pile-up around the tip during nanoindentation. The contact depth $h_{c}$ then strongly differs from the total penetration depth $h$ (which is the depth reached by the tip from the initial specimen surface).

The standard methods widely used to extract contact area require analytical models based on material rheology assumptions, which use penetration depth and contact stiffness values to compute the contact depth $[18,19]$. On the contrary, the direct monitoring of contact area by resistive-nanoindentation brings supplementary inputs to the quantitative analysis of indentation tests. This is expected to be an experimental alternative to standard analytical models.

A first attempt to extract mechanical contact area from electrical measurements was reported by Fang et al [14] for micro-indentation. The authors successfully related non-linear current-bias curves performed during micro-indentation to real-time contact area. However, this approach is inadequate for nanoindentation. More promising results were obtained by Sprouster et al [15], with resistive nanoindentation performed on a gold bulk sample with a vanadium carbide tip. The measured resistance during indentation was successfully fitted using an analytical model based on Maxwell expression for the spreading resistance (Eq. (4), with $\rho$ the material resistivity and $r_{c}$ the contact radius). With this method, the authors managed to compute their specimen resistivity but the ability to monitor the contact area during nanoindentation was not discussed.

$$
R_{\text {spreading }}=\frac{\rho}{4 r_{c}}
$$

In the present work, a complete method is proposed to compute contact area using resistivenanoindentation of noble metals. First, the different contributions to the measured resistance are discussed, then the computation procedure is detailed and applied step-by-step: (1) measurement of the tip shape, (2) set-up calibration on a model specimen (gold bulk single crystal) and (3) extraction of the contact area on a specimen with complex rheology (gold film on sapphire substrate), and finally, the data generated by this method are compared to experimental AFM measures of contact areas. 


\section{Experimental details}

Nanoindentation testing is performed with a home-made device, which includes a commercial actuator from Nanomechanics Inc. Resistance measurements are conducted with a ResiScope apparatus from Scientec [20]. Nanoindentation tests are performed with a Berkovich boron-doped diamond (BDD) tip with resistivity in the range [0.2-2] $\Omega . \mathrm{cm}$. Resistive-nanoindentation tests were performed on a gold bulk single crystal (100) for the calibration step, and the whole method was validated on a $200 \mathrm{~nm}$-thick polycrystalline gold film deposited on a sapphire substrate. Gold samples were chosen as model specimens, due to the absence of native oxide. Nonetheless this method can be applied to any oxide-free highly-conductive material. Indentation of a composite geometry such as an elasto-plastic film (gold) on an elastic substrate (sapphire) is a model case of complex rheology which is depth-dependent and where no analytical modeling exists. AFM imaging of residual imprints is performed after each test for validation purpose. Post-mortem contact areas are computed using Gwyddion software [21]: a map of local plane inclination angles is first extracted from the topological map of these imprints [22]. Then manually-adjusted edge detection is performed on the obtained map (standard deviation on contact area lower than 3\%). AFM images are performed after the final elastic unloading (viscous relaxation is negligible at room temperature): this leads to a small modification of the indentation imprint, as compared to the contact area under loading. According to Charleux et al. [22], the induced error on contact area is lower than $10 \%$. 


\section{Results and discussion}

\section{Resistive-nanoindentation curves}

The resistance-versus-depth curves obtained during the indentation of the gold bulk sample on different spots are given in Fig. 1(a). This figure shows the high reproducibility of these experiments. As expected, the resistance continuously decreases as the mechanical contact area increases [15]. Fig. 1(b) shows another set of resistive-nanoindentation tests performed at different polarization biases. One can notice that the resistance is bias-independent, which is the evidence of an ohmic-like electrical contact, in agreement with the absence of any interfacial layer between the tip and specimen.
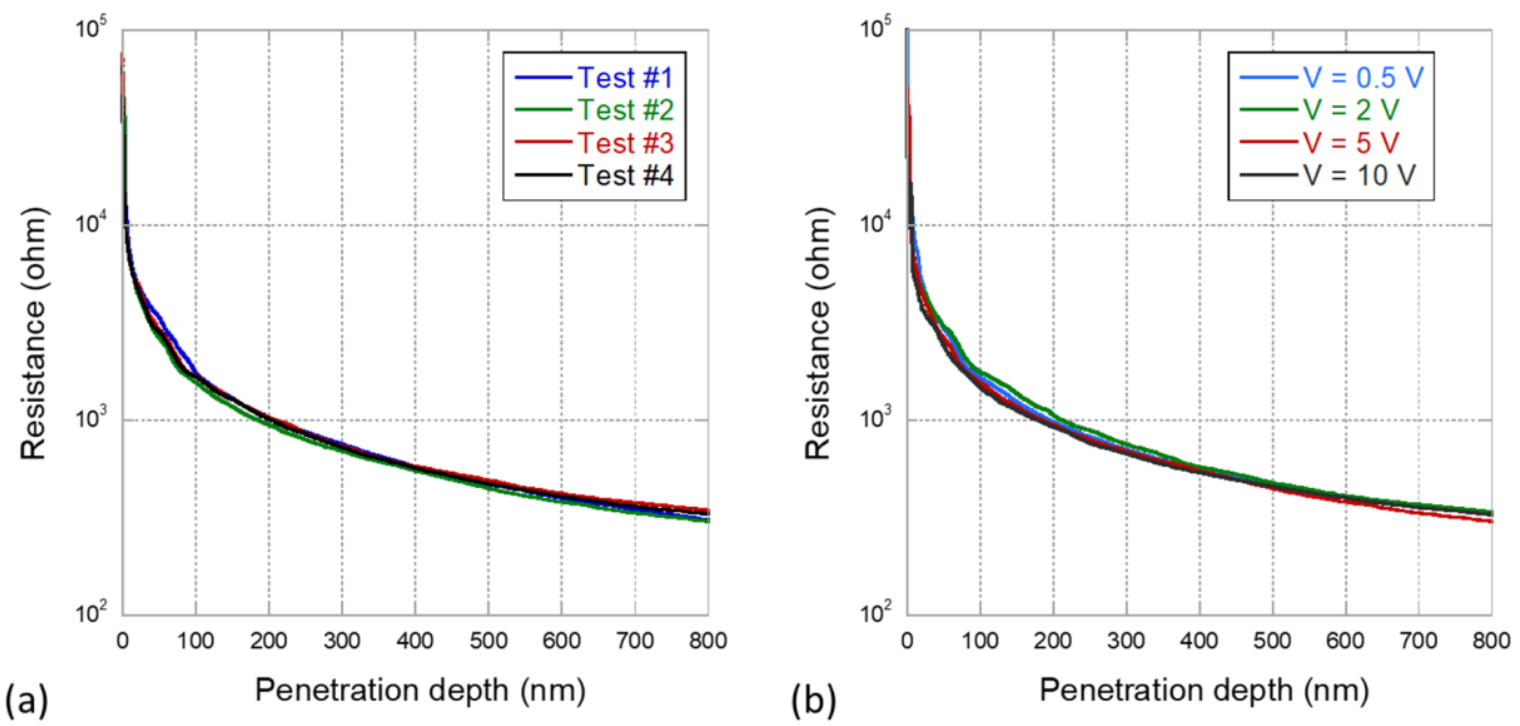

FIG. 1. Resistance evolution during indentation of gold bulk single crystal. (a) Different tests performed at fixed polarization bias (5V) and (b) performed at different polarization biases. $<<$ color online $>>$ 


\section{Identification of resistance contributions}

Before starting the procedure for the contact area computation, the different contributions to the measured resistance have to be identified. As long as the electronic transport between the BDD tip and the gold specimen remains in diffusive regime, the measured resistance is the sum of the following contributions (from top to bottom in Fig. 2):

i. The electrical resistance of the indentation tip $\left(R_{\text {tip }}\right)$;

ii. An interface resistance $\left(R_{\text {interface }}\right)$, coming from a possible interfacial layer;

iii. The spreading resistance within the sample ( $\left.R_{\text {spreading-sample }}\right)$, due to the current line constriction at the contact;

iv. The resistance from the gold sample $\left(R_{\text {sample }}\right)$;

v. An additional series resistance from the experimental set-up $\left(R_{\text {series }}\right)$.

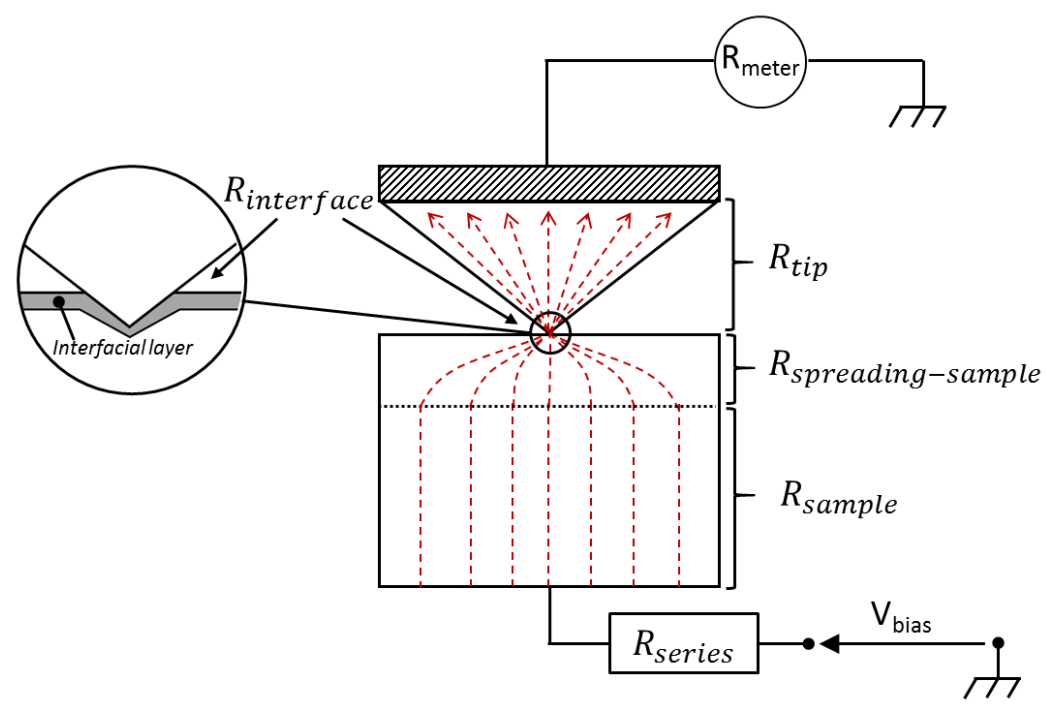

FIG. 2. Contributions to the measured resistance. Current lines are schematized by red-dotted lines. 
The two resistance contributions coming from the gold sample ( $R_{\text {spreading-sample }}$ and $R_{\text {sample }}$ ) depend linearly on its resistivity, similarly to the tip resistance $\left(R_{t i p}\right)$ that depends linearly on the BDD tip resistivity. Since gold resistivity is at least five orders of magnitude lower than the BDD tip resistivity, $R_{\text {sample }}$ and $R_{\text {spreading-sample }}$ can be safely neglected. $R_{\text {interface }}$ can also be neglected, due to the absence of oxide or of other contaminant layer at the tip-to-sample interface. Consequently, the measured resistance $R_{\text {measured }}$ is simply the sum of the tip resistance and the additional constant series resistance:

$$
R_{\text {measured }}=R_{\text {series }}+R_{\text {tipp }}
$$

The expression for $R_{t i p}$ can be adapted from a model proposed by Schneegans et al for the computation of a broken AFM tip [23]: the broken apex of the AFM tip is, in our case, the part of the indentation tip which has penetrated the sample (Supplementary Fig. S2(a)). This consideration leads to Eq. 6 (with $\rho_{\text {tip }}$ the tip resistivity and $\alpha$ a constant related to the tip geometry):

$$
R_{\text {tip }}=\alpha \cdot \frac{\rho_{\text {tip }}}{h_{c}}
$$

However the experimental determination of $h_{c}$ is affected by the unavoidable tip defect (rounded apex of height $h_{0}$ (Supplementary Fig. S2(b)), thus Eq. 6 turns into Eq. 7:

$$
R_{\text {tip }}=\alpha \cdot \frac{\rho_{\text {tip }}}{h_{c}+h_{0}} \quad(7)
$$

This expression relies on two strong assumptions: 1 / the current distribution within the tip is homogeneous and parallel to the tip axis and $2 /$ the part of the tip inside the sample affects only linearly the tip resistance.

Regarding the first assumption, it has been shown in the literature that the error arising from the use of a homogeneous and parallel distribution of current lines within the tip is less than $10 \%$ [24]. The second assumption is more questionable. Since the BDD tip is five orders of magnitude more resistive than gold, current lines are expected to be localized at the periphery of the contact instead of being homogeneously 
distributed (Supplementary Fig. S3). This point has been confirmed by numerical computation (not shown). In literature, Nakamura et al [25] have shown that the electrical resistance of ring contacts is equivalent to the resistance of the corresponding full-surface contact divided by a shape factor $S F$. This shape factor depends on the ratio between the ring thickness and the contact radius. For now, we first assume that this shape factor $S F$ is constant during indentation (this assumption will be confirmed later).

Finally, the measured resistance is given by Eq. 8, where A and B indicate two constants that depend only on the experimental set-up (series resistance, tip geometry and resistivity) but not on the specimen. These constants have to be determined experimentally during the calibration step (below), and will be further used for the effective nanoindentation measurements.

$$
R_{\text {measured }}=A+\frac{B}{h_{C}+h_{0}}
$$

\section{Procedure for contact area computation}

Now that these preliminary considerations have been stated, the step-by-step procedure for contact area computation can be described:

1. First the BDD tip geometry is determined. This step generates the tip "shape function" that relates the projected contact area to the contact depth.

2. An electrical calibration is carried out in order to determine the A and B constants (Eq. 8). As A and B depend only on the experimental set-up, a one-to-one analytical correspondence can be established between electrical resistance and contact depth (independently of the specimen).

3. The characterisation of a conductive oxide-free specimen can thus be performed. Using the shape function (step 1), the contact area is finally determined from the contact depth values (step 2) for this specimen to be characterized.

Step 1. The determination of the tip geometry allows us to relate the total penetration depth $h$ to the corresponding tip cross section $A$. For that purpose, two complementary methods have been used: direct 
AFM imaging of the tip, and the calibration method described by Oliver and Pharr (where a model specimen with known isotropic elastic properties, like fused $\mathrm{SiO}_{2}$, is measured) [17]. The obtained shape function can be simplified, leading to Eq. 9. The error due to this simplification is less than 5\% for penetration depth larger than $45 \mathrm{~nm}$.

$$
A_{c}=\pi\left(\alpha_{\text {tip }}\left(h_{c}+h_{0}\right)\right)^{2} ; \alpha_{\text {tip }}=2.67 ; h_{0}=11.6 \mathrm{~nm}
$$

Step 2. In this work, electrical calibration has been performed on a gold bulk sample. Several resistive nanoindentation tests were run, up to different contact depths (circa 100, 300, 600 and $800 \mathrm{~nm}$ ). Postmortem AFM imaging shows that residual imprints exhibit sink-in, probably due to polishing-induced surface hardening (Fig. 3(a)). Due to sink-in evidence, the contact depth can be reliably estimated from Oliver and Pharr model [18]. In order to validate the use of this model, the contact area was also computed from contact depth values, thanks to the tip "shape function" determined at step 1 . The obtained contact area values were then compared to post-mortem AFM imaging, as shown in Fig. 3(a). This figure shows very good agreement between the estimated contact area using Oliver and Pharr model and the postmortem AFM measurements. This step allows us to relate the total penetration depth to the contact depth. 


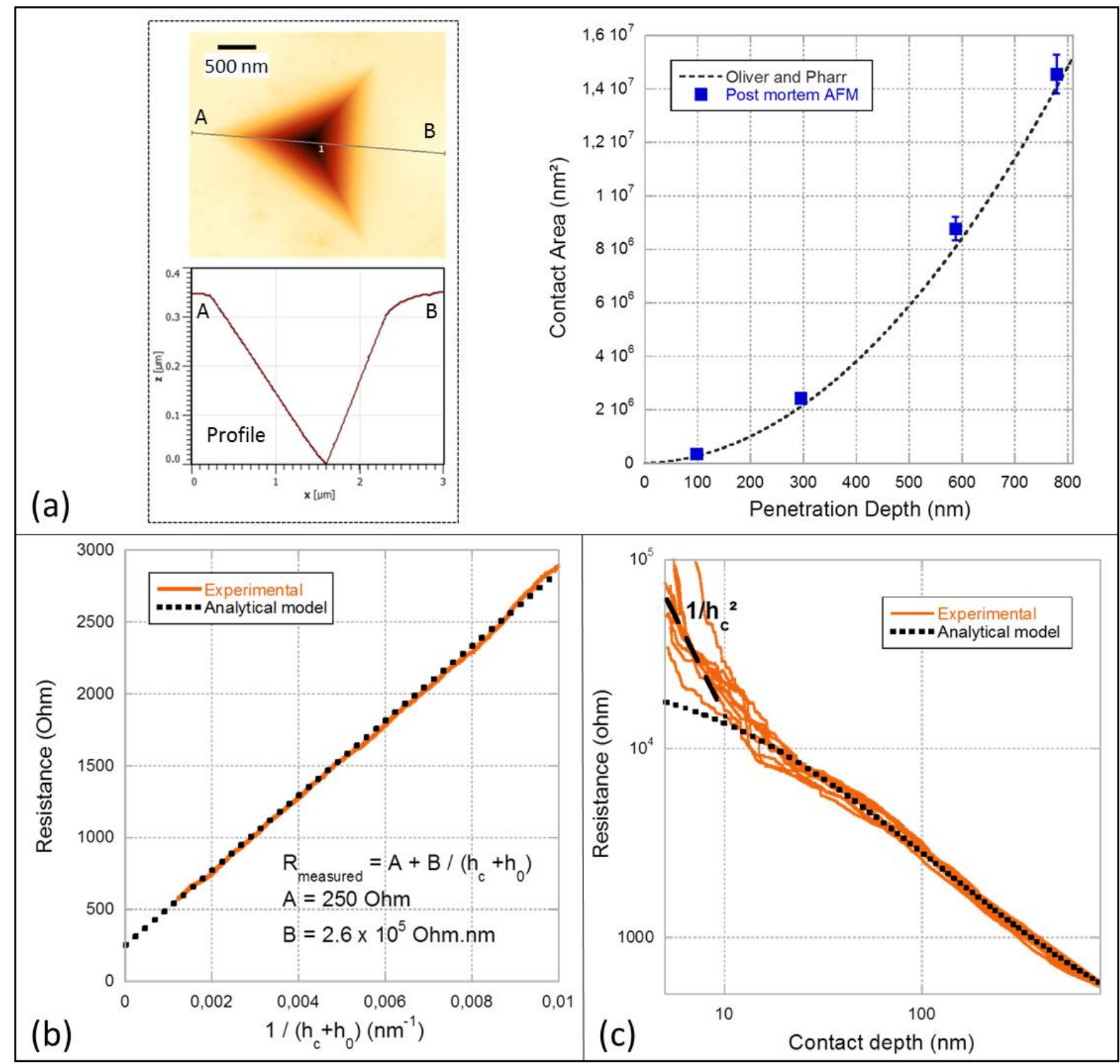

FIG. 3. Results from resistive-nanoindentation on bulk gold sample. (a) Post-mortem AFM measures (left) and contact area against penetration depth (comparison of the Oliver-Pharr model predictions to the post-mortem AFM measures, right). (b) Fitting of the resistance evolution against the reciprocal of the corrected contact height. (c) Resistance-versus-depth curves in logarithmic scales.

At this point, it is possible to plot the measured resistance $R_{\text {measured }}$ versus $1 /\left(h_{c}+h_{0}\right)$ (Fig. 3(b)) so that Eq. 8 can be fitted. The linear correlation (coefficient of 0.99) validates the proposed analytical 
expression of the measured resistance (Eq. 8). This linearity also validates the assumption of a constant shape factor $S F$ during indentation. A and B constants are then obtained by simple linear fitting. Using these fitted values, both experimental and analytical resistance versus depth curves can be plotted (Fig. 3(c)). Remarkably, a very good agreement is found between the experimental results and the analytical model for contact depths larger than $20 \mathrm{~nm}$. Below these depths, the measured resistance is much higher than the model prediction. This deviation is most likely due to a change in electronic transport regimes from diffusive to ballistic since in ballistic regime, the electrical resistance evolves as $1 / h_{c}{ }^{2}$ [26]. This trend is in good agreement with that observed experimentally at small depths (Fig. 3(c)) Alternatively, this deviation could also be explained by a multi-contact mode [27], due to a potential thin interfacial layer at the contact.

Step 3. Once the entire analytical expression is fully set from the calibration sample, the specimen to be characterized can be processed: contact depth can then be directly determined from the resistance measurements. In the present work, this has been applied to a $200 \mathrm{~nm}$ polycrystalline gold thin film on sapphire substrate. This sample has a complex rheology, as the film thickness acts as an additional length scale. The deformation field during nanoindentation is no more self-similar as in the bulk case. The standard practice to analyze such a soft-film-on-stiff-substrate structure is usually to determine the contact area by numerically solving a Fredholm integral [28] (or using finite elements simulations) coupled with at least one post-mortem measurement of the remaining imprints at a given depth (by SEM or AFM).

\section{Experimental validation}

Resistive-nanoindentation tests with various final penetration depths were performed on this gold thin film. For each test, the contact depths were extracted continuously from resistance measurements (using Eq. 8) and the corresponding contact areas were computed from the tip shape function (using Eq. 9). After each test, post-mortem AFM measurements were conducted for validation. Fig. 4 reports the contact areas extracted from this procedure as well as post-mortem imaging data. An excellent agreement is observed, thus validating the whole methodology. It can be seen that the deformed film at large relative penetration 
depth $(75 \%)$ presents a large upheaval. At this depth the contact area extracted from the Oliver-Pharr's method [18] (based on sink-in assumption) would be $\sim 50 \%$ lower. The present procedure should apply to any specimen that satisfies the two following conditions: (1) the specimen is much more conductive than the indentation tip and (2) the specimen is oxide-free. The first condition is easily met for bulk metals, while the relevant magnitude is the sheet resistance for thin films. In the case of oxidized specimens, several potential solutions might be envisaged but require further assessments: application of large-enough voltages leading to dielectric breakdown, measurement under controlled environment (controlled relative humidity, submersion in liquids,...), tuning of applied voltages in accordance with the electrochemical potentials of the involved materials,...

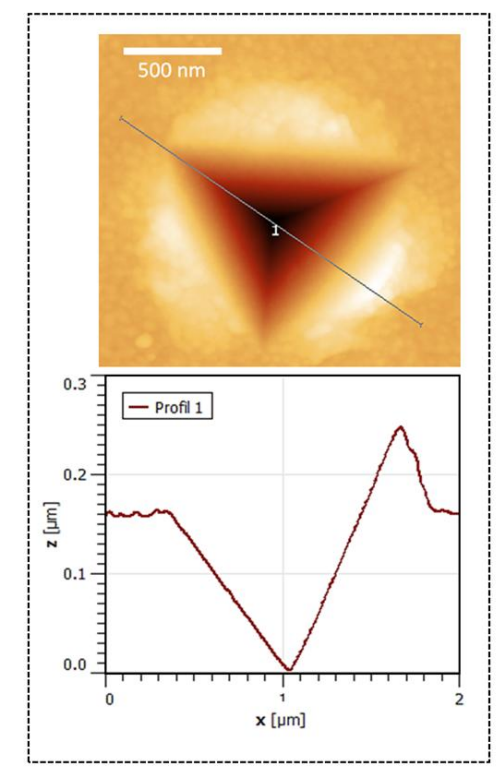

(a)

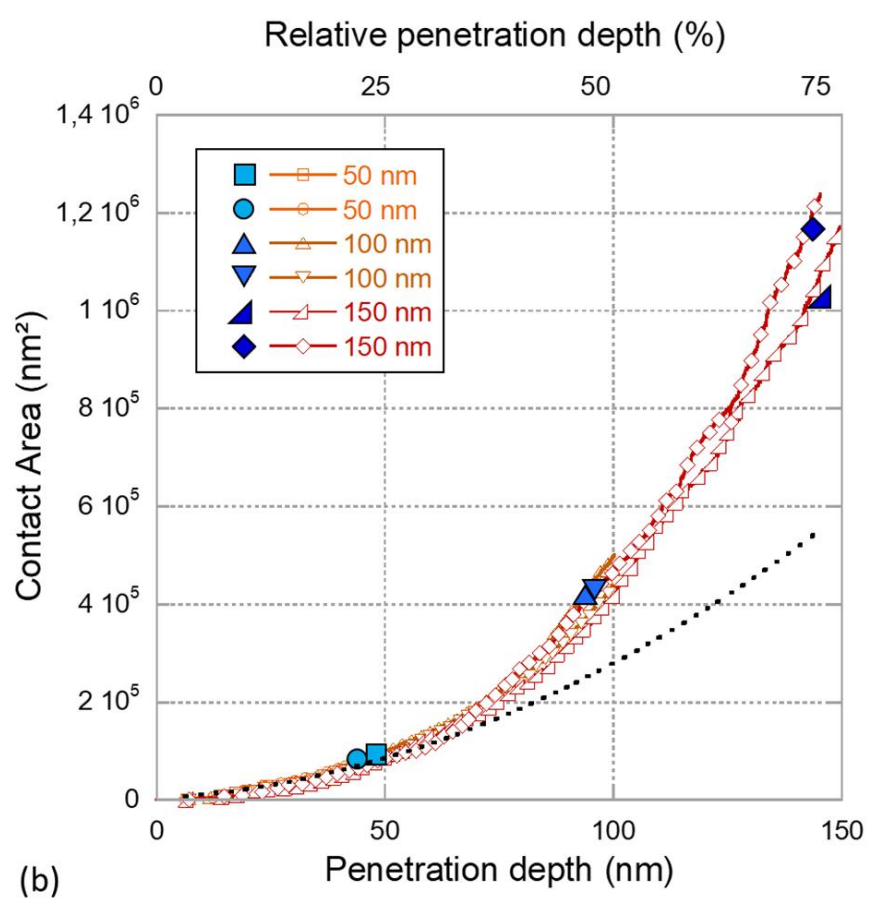

FIG. 4. Analysis of the resistive-nanoindentation tests on the $200 \mathrm{~nm}$ gold thin film. (a) Post-mortem AFM image and AFM profile showing evidence of pile-up. (b) Contact area against penetration depth: comparison of the data computed from the electrical measurements (open markers) and the corresponding post-mortem measurements (solid markers). Contact area from Oliver and Pharr model in dotted line. 


\section{Conclusion}

This paper reports an efficient method to extract the contact area during nanoindentation from resistance

measurements. This method relies on three steps (tip shape measurement, set-up calibration, application to the specimen under test), and applies to oxide-free conductive materials. In the presence of an oxide layer, an additional non-linear contact resistance is expected, thus requiring the adaptation of the approach presented in this paper. Finally, the method is applied to nanoindentation tests performed on a sample with a complex rheology. The excellent agreement with the experimental area determination validates the proposed method. To our knowledge, this is the first time in literature that the contact area is extracted from electrical measurements during nanoindentation. It also paves the way for new calibration procedure of both tip geometry and frame stiffness.

\section{Acknowledgments}

This work has been performed with the financial support of the Centre of Excellence of Multifunctional Architectured Materials "CEMAM" $n^{\circ}$ ANR-10-LABX-44-01. The CEMAM program is funded by the French Agence Nationale de la Recherche (ANR).

The authors thank the technical team of SIMaP lab (Bruno Mallery, Stéphane Massucci and Nadine Vidal) for its support, as well as members of CSI/Scientec company (Les Ulis, France) for their support for the functionalisation of the nanoindentation head: Louis Pacheco, Alan Lecoguiec and Sylvain Poulet. 


\section{Supplementary Material Available}

Schematics describing the material rheology, the tip geometry and the current line distribution are available as supplementary material.

FIG. S1. Effect of material rheology on contact area and contact depth. The penetration depth $h$ is the depth reached by the tip from the initial specimen surface, while the contact depth $h_{c}$ describes the length of the tip in contact with the specimen.

FIG. S2. Schematics of the considered tips with the corresponding dimensions. (a) Defect-free tip. (b) Real tip with rounded apex.

FIG. S3. Current line distribution (red-dotted lines and surfaces) for a Berkovich tip. The ideal case where the current lines are homogeneously distributed is compared to the more realistic case, where current lines are localized at the contact periphery. Plane-view and cross-section views are shown. 


\section{References}

1. H. Nili, K. Kalantar-Zadeh, M. Bhaskaran and S. Sriram: In situ nanoindentation: Probing nanoscale multifunctionality. Prog Mater Sci. 58, 1 (2013)

https://doi.org/10.1016/j.pmatsci.2012.08.001

2. D. R. Clarke, M. Kroll, P. D. Kirchner, R. F. Cook and B. J. Hockey: Amorphization and Conductivity of Silicon and Germanium Induced by Indentation. Phys. Rev. Lett. 60, 2156 (1988)

https://doi.org/10.1103/PhysRevLett.60.2156

3. G. M. Pharr, W. C. Oliver, R. F. Cook, P. D. Kirchner, M. Kroll and T. R. Dinger: Electrical resistance of metallic contacts on silicon and germanium during indentation. J. Mater. Res. 7, 961 (1992)

https://doi.org/10.1557/JMR.1992.0961

4. A. B. Mann, D. van Heerden, J. B. Pethica and T. P. Weihs: Size-dependent phase transformation during point loading of silicon. J. Mater. Res. 15, 1754 (2000)

https://doi.org/10.1557/JMR.2000.0253

5. A. B. Mann, D. van Heerden, J. B. Pethica, P. Bowes and T. P. Weihs: Contact resistance and phase transformation during nanoindentation of silicon. Philos. Mag. A 82, 1921 (2002)

https://doi.org/10.1080/01418610208235704

6. J. E. Bradby, J. S. Williams and M. V. Swain: In situ electrical characterisation of phase transformation in Si during indentation. Phys. Rev. 67, 085205 (2003)

https://doi.org/10.1103/PhysRevB.67.085205 
7. S. Ruffell, J. E. Bradby, J. S. Williams and O. L. Warren: An in situ electrical measurement technique via a conducting diamond tip for nanoindentation in silicon. J. Mater. Res. 22, 578 (2007)

https://doi.org/10.1557/jmr.2007.0100

8. S. Ruffell, J. E. Bradby, N. Fujisawa and J. S. Williams: Identification of nanoindentation-induced phase changes in silicon by in situ electrical characterization. J. Appl. Phys. 101, 083531 (2007)

https://doi.org/10.1063/1.2724803

9. J. B. Pethica and D. Tabor: Contact of characterised metal surface at very low loads: deformation and adhesion. Surf. Sci. 89, 189 (1979)

https://doi.org/10.1016/0039-6028(79)90606-X

10. D. D. Stauffer, R. C. Major, D. Vodnick, J. H. Thomas, J. Parkern, M. Manno, C. Leighton and W. W. Gerberich: Plastic response of the native oxide on $\mathrm{Cr}$ and $\mathrm{Al}$ thin films from in situ conductive nanoindentation. J. Mater. Res. 27, 685 (2012)

https://doi.org/10.1557/jmr.2011.432

11. H. H. Nguyen, P. J. Wei and J. F. Lin: Electrical contact resistance for monitoring nanoindentationinduced delamination. Adv. Nat. Sci.: Nanosci. Nanotechnol. 2, 015007 (2011)

https://doi.org/10.1088/2043-6262/2/1/015007

12. P. Y. Duvivier, V. Mandrillon, K. Inal, C. Dieppedale, S. Deldon-Martoscia and J. P. Polizzi: Time dependence investigation of the electrical resistance of $\mathrm{Au} / \mathrm{Au}$ thin film micro contacts. Proc. 56th IEEE Holm Conf. Elect. Cont. 58 (2010)

https://doi.org/10.1109/HOLM.2010.5619563 
13. B., Arrazat, P. Y. Duvivier, V. Mandrillon and K. Inal: Discrete analysis of gold surface asperities deformation under spherical nano-indentation towards electrical contact resistance calculation. Proc. 57th IEEE Holm Conf. Elect. Cont. 1, (2011)

https://doi.org/10.1109/HOLM.2011.6034798

14. L. Fang, C. L. Muhlstein, J. G. Collins, A. L. Romasco and L. H. Friedman: , Continuous electrical in situ contact area measurement during instrumented indentation. J. Mater. Res. 23, 2480 (2008)

https://doi.org/10.1557/jmr.2008.0298

15. D. J. Sprouster, S. Ruffel, J. E. Bradby, D. D. Stauffer and R. C. Major, O. L. Warren, J. S. Williams: Quantitative electromechanical characterization of materials using conductive ceramic tips. Acta Mater.

$71,153(2014)$

https://doi.org/10.1016/j.actamat.2014.02.028

16. I. N. Sneddon: The relation between load and penetration in the axisymmetric boussinesq problem for a punch of arbitrary profile. Int. J. Eng. Sci., 3, 47 (1965)

https://doi.org/10.1016/0020-7225(65)90019-4

17. W. C. Oliver and G. M. Pharr: Measurements of hardness and elastic modulus by instrumented indentation: Advances in understanding and refinements to methodology. J. Mater. Res. 19, 3 (2004) http://dx.doi.org/10.1557/jmr.2004.0002

18. W. C. Oliver and G. M. Pharr: An improved technique for determining hardness and elastic modulus using load and displacement sensing indentation experiments. J. Mater. Res. 7, 1564 (1992)

https://doi.org/10.1557/JMR.1992.1564

19. J. L. Loubet, M. Bauer, A. Tonck, S. Bec and B. Gauthier-Manuel: Mechanical Properties and Deformation Behavior of Materials Having Ultra-Fine Microstructures : Nanoexperiments with a surface 
force apparatus, in Mechanical Properties and Deformation Behavior of Materials Having Ultra-Fine Microstructures, edited by M. Nastasi, D. M. Parkin and H. Gleiter, Kluwer Academic Publisher, 429 (1993)

https://doi.org/10.1007/978-94-011-1765-4

20. F. Houzé, R. Meyer, O. Schneegans and L. Boyer: Imaging the local electrical properties of metal surfaces by atomic force microscopy with conducting probes. Appl. Phys. Lett. 69, 1975 (1996)

https://doi.org/10.1063/1.117179

21. Gwyddion software. Available at : http://gwyddion.net/download.php (accessed 14 May 2019).

22. L. Charleux, V. Keryvin, M. Nivard, J.-P. Guin, J.-C. Sangleboeuf, Y. Yokoyama: A method for measuring the contact area in instrumented indentation testing by tip scanning probe microscopy imaging, Acta Mat. 70, 249-258 (2014)

https://doi.org/10.1016/j.actamat.2014.02.036

23. O. Schneegans, P. Chrétien, F. Houzé and R. Meyer: Capacitance measurements on small parallel plate capacitors using nanoscale impedance microscopy. Appl. Phys. Lett. 90, 043116 (2007)

https://doi.org/10.1063/1.2437052

24. J. D. Romano: The conical resistor conundrum: a potential solution. R. H. Price, Am. J. Phys., 64, 1150 (1996)

https://doi.org/10.1119/1.18335

25. M. Nakamura: Constriction resistance of conducting spots by the boundary element method. IEEE Trans. Comp. Hybrids, Manufact. Technol. 16, 339 (1993)

https://doi.org/10.1109/33.232062

26. Y. Sharvin: A possible method for studying Fermi surfaces. Sov. Phys. JETP 21, 655 (1965) 
27. J. A. Greenwood: Constriction resistance and the real area of contact. J. Appl. Phys. 17, 1621 (1966) https://doi.org/10.1088/0508-3443/17/12/310

28. A. Perriot and E. Barthel: Elastic contact to a coated half-space - Effective elastic modulus and real penetration. J. Mater. Res. 19, 600 (2004)

https://doi.org/10.1557/jmr.2004.19.2.600 


\section{Figure captions}

FIG. 1. Resistance evolution during indentation of gold bulk single crystal. (a) Different tests performed at fixed polarization bias (5V) and (b) performed at different polarization biases. <<color online>>

FIG. 2. Contributions to the measured resistance. Current lines are schematized by red-dotted lines.

FIG. 3. Results from resistive-nanoindentation on bulk gold sample. (a) Post-mortem AFM measures (left) and contact area against penetration depth (comparison of the Oliver-Pharr model predictions to the post-

mortem AFM measures, right). (b) Fitting of the resistance evolution against the reciprocal of the corrected contact height. (c) Resistance-versus-depth curves in logarithmic scales.

FIG. 4. Analysis of the resistive-nanoindentation tests on the $200 \mathrm{~nm}$ gold thin film. (a) Post-mortem AFM image and AFM profile showing evidence of pile-up. (b) Contact area against penetration depth: comparison of the data computed from the electrical measurements (open markers) and the corresponding post-mortem measurements (solid markers). Contact area from Oliver and Pharr model in dotted line. 